A GUIDE TO BIOMOLECULAR SIMULATIONS 


\section{FOCUS ON STRUCTURAL BIOLOGY}

Volume 4

Series Editor

ROB KAPTEIN

Bijvoet Center for Biomolecular Research, Utrecht University, The Netherlands 


\title{
A Guide to Biomolecular Simulations
}

\author{
by \\ OREN M. BECKER \\ Predix Pharmaceuticals, Ramat-Gan, Israel \\ and \\ MARTIN KARPLUS \\ Harvard University, Cambridge, MA, U.S.A. and \\ Université Louis Pasteur, Strasbourg, France
}


A C.I.P. Catalogue record for this book is available from the Library of Congress.

ISBN-10 1-4020-3586-1 (HB)

ISBN-10 1-4020-3587-X (e-book)

ISBN-13 978-1-4020-3586-9 (HB)

ISBN-13 978-1-4020-3587-6 (e-book)

Published by Springer,

P.O. Box 17, 3300 AA Dordrecht, The Netherlands.

www.springer.com

Printed on acid-free paper

All Rights Reserved

(C) 2006 Springer

No part of this work may be reproduced, stored in a retrieval system, or transmitted in any form or by any means, electronic, mechanical, photocopying, microfilming, recording or otherwise, without written permission from the Publisher, with the exception of any material supplied specifically for the purpose of being entered and executed on a computer system, for exclusive use by the purchaser of the work.

Printed in the Netherlands. 
Preface

Introduction: Note to the Student $\quad 1$

Introduction: Note to the Instructor 3

Introduction: UNIX 4

Introduction: CHARMM Primer $\quad 10$

Introduction: $\quad$ CHARMM Template Files 18

Lab 1: $\quad$ Introduction to Molecular Visualization $\quad 19$

Lab 2: $\quad$ Energy and Minimization $\quad 35$

Lab 3: $\quad$ Minimization and Analysis $\quad 51$

Lab 4: Conformational Analysis $\quad 65$

Lab 5: $\quad$ Basic Molecular Dynamics in Vacuum and in Solution 75

Lab 6: $\quad$ Molecular Dynamics and Analysis 101

Lab 7: $\quad$ Ligand Dynamics in Myoglobin 131

Lab 8: $\quad$ Normal Mode Analysis 155

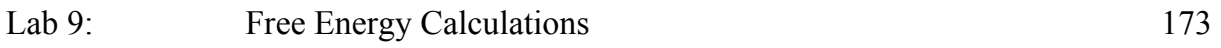

Lab 10: $\quad$ Minimum Energy Paths 193

Lab 11: Multiple Copy Simultaneous Search 205

Lab 12: $\quad$ Hemoglobin Cooperativity: the T-R Transition 213 
The first molecular dynamics simulation of a macromolecule of biological interest was published in 1977. The simulation concerned the bovine pancreatic trypsin inhibitor (BPTI), which has served as the "hydrogen molecule" of protein dynamics because of its small size, high stability and relatively accurate x-ray structure, available in 1975; interestingly, its physiological functions remain unknown. Although this simulation was crude by present-day standards, the results were instrumental in replacing our view of proteins as relatively rigid structures with the realization that they were dynamic systems, whose internal motions play a functional role. Over the intervening years, such simulations have become a central part of biophysics.

Simulations can provide the ultimate detail concerning individual particle motions as a function of time, so they can be used to answer specific questions about the properties of a model system, often more easily than experiments on the actual system. A significant aspect of simulations is that, although the potentials employed in simulations are approximate, they are completely under the user's control, so that by removing or altering specific contributions their role in determining a given property can be examined. This is most graphically demonstrated by the use of 'computer alchemy' - transmuting the potential from that representing one system to another during a simulation - in the calculation of free energy differences.

The growth in the number of studies using molecular dynamics for biomolecular simulations has been fueled by the general availability of programs and the computing power required for meaningful studies. The first simulation was less than 10 ps in lengths, but it is now de rigueur to do simulations that are at least 1000 times as long (10 ns); they, nevertheless, take a factor of about 50 less time for the same sized system, though of course much larger systems (104 to 106 atoms instead of 500) are sometimes necessary to study if an explicit solvent and/or membrane environment is included. Another important consequence of the access to faster computers is the fact that multiple simulations can be done to obtain converged results with statistical error estimates.

The number of publications using molecular dynamics is now in the thousands (A search with "molecular dynamics" and "proteins" as key words of the ISI "Web of Science" [http://www.isinet.com/isi/products/ 
citation/wos/] yielded 2337 papers published since the beginning of the new millenium (January 2000).),

Applications of molecular dynamics in biophysics range over many areas. They are used in the structure determination of macromolecules with x-ray and NMR data, the modelling of unknown structures from their sequence, the study of enzyme mechanisms, the estimation of ligandbinding free energies, the evaluation of the role of conformational change in protein function, and drug design for targets of known structures.

The widespread application of molecular dynamics and related methodologies suggests that it would be useful to have available an introductory self-contained course by which students with a relatively limited background in chemistry, biology and computer literacy, can learn the fundamentals of the field. This "A Guide to Biomolecular Simulations" tries to fill this need. As the basic learning tool, the exercises in the Guide are based on the CHARMM program, which is one of the most widely used programs in the field. CHARMM was selected because it is the program with which we do our research. To graphically illustrate the results we have used a version of QUANTA, which is distributed by Accelrys, Inc.

The Guide consists of six chapters which provide the fundamentals of the field and six chapters which introduce the reader to more specialized but important applications of the methodology. For the latter, a selection was made, based primarily on our experience.

We hope that students who work through this Guide will be able to go on to read papers in the field with a good level of understanding and to initiate their own studies of fundamental and applied problems in the rapidly growing area of molecular dynamics simulations. References are given, many to the original work in a given area. Using them as a key, the student will be able to search for more recent work on the Web.

Many people have contributed to making this Guide a reality. We thank them in the Acknowledgements. 


\section{ACKNOWLEDGEMENTS}

The material on which the Guide is based was developed at Harvard University during the 1990's over several years as a companion "laboratory" for the biophysics course "Molecular Biophysics Simulations of Macromolecules" (Biophysics 164), the lectures of which were given mainly by one of the authors. The original course did not have a laboratory and was taught jointly by Steve Harrison and Martin Karplus. After the latter became responsible for the course in its entirety, a "laboratory" was introduced in which students were able to do computer calculations that illustrated the points made in the lectures.

The first version of the laboratory manual was prepared by Herman van Vlijmen, based on the exercises developed by Postdoctoral Fellows from the Karplus group. Many of the original contributions were extended and modified over the years. The list of the original contributors:

Lab 1: Herman van Vlijmen, Oren M. Becker, Yael Marantz

Lab 2: Diane Joseph-McCarthy, Carla Mattos

Lab 3: Annick Dejaegere, Roland Stote

Lab 4: Annick Dejaegere, Roland Stote

Lab 5: Oren M. Becker

Lab 6: Jeffrey D. Evanseck, Masakatsu Watanabe, Oren M. Becker

Lab 7: Leo S. D. Caves

Lab 8: Oren M. Becker

Lab 9: Stefan Boresch, Oren M. Becker

Lab 10: Oren M. Becker

Lab 11: Amedeo Caflisch

Lab 12: Leo S. D. Caves

We would also like to thank Ryan Bitetti-Putzer, Aaron Dinner, Carla Mattos, Sung-Sau So, Herman van Vlijmen and Ori Kalid for reading through the Guide, testing it, and offering valuable comments. 